\title{
ESTUDIO EPIDEMIOLÓGICO EN UNA MUESTRA CLÍNICA DE MUJERES A TRAVÉS DEL DIS
}

\author{
López-García, G.', Cortegano, M.C. ${ }^{1}$, Martín, C.', Murcia, L.', López-Mora, I.', \\ García Montalvo, C. ${ }^{2}$ y López Soler, C. ${ }^{2}$ \\ 1 Unidad de Psicologia Clinica Hospital "Virgen de La Arrixaca" de Murcia. \\ 2 Universidad de Murcia
}

\begin{abstract}
RESUMEN
La investigación epidemiológica llevada a cabo en las últimas décadas en nuestro país ha demostrado, de manera inequivoca, la existencia de marcadas diferencias en la morbilidad psicopatológica y en el patrón de conducta de enfermedad desarrollados según el sexo (Vazquez-Barquero, Díez Manrique, Peña, Quintanal y Labrador López, 1986; Herrera, Antonell, Espagnolo, Domenech y Martín, 1987; Herrera, Antonell, Spagnolo y Gispert, 1990). Este hecho, unido a la constatación de que la prevalencia psicopatológica en la mujer es mucho mayor que en el hombre y que el riesgo de padecer un trastorno mental puede ser casi del doble en el sexo femenino (Muñoz, 1979; Herrera et al., 1987; Vazquez-Barquero, Diez Manrique y Peña, 1987), nos hacen plantear la presente investigación con el objetivo de conocer el tipo de desórdenes psicológicos que afectan de forma preferente a la población femenina, objetivo éste enmarcado en un planteamiento metodológico de tipo epidemiológico descriptivo
\end{abstract}

Palabras clave: modelo de entrevista de diagnóstico, psicopatología, epidemiología, trastornos mentales, mujeres

\begin{abstract}
The epidemiologic investigation made in our country during last decades has shown, unequivocaly, the existence of prononced diferences in the psychopatologic morbility and in the disease model of behaviour developed depending on the sex (VazquezBarquero, Díez Manrique, Peña, Quintanal y Labrador López, 1986; Herrera, Antonell, Espagnolo, Domenech y Martín, 1987; Herrera, Antonell, Spagnolo y Gispert, 1990). This fact joint to the prove that woman psychopatologic prevail is bigger than man one and the risk of having a mental disorder can be almost the double in the female sex (Muñoz, 1979; Herrera et al., 1987; Vazquez-Barquero, Diez Manrique, y Peña, 1987), make us to establish the present investigation in order to know the kind of psychological disorders wich affect on preferent way to female population, wich is the objetive on the methodological planning of descriptive epidemiological king
\end{abstract}

Key words: diagnostic interview schedule, psychopathology, epidemiology, mentaldisorders, women

Correspondencia: Carmen Garcia Montalvo. Departamento de Personalidad, Evaluación y Tratamiento psicológicos. Facultad de Psicologia. Edificio Luis Vives. Campus de Espinardo s/n. 30071, Murcia.Tfo: 363447.Fax: 364115 


\section{INTRODUCCIÓN}

El objetivo primordial que guia la realización del presente trabajo de investigación es el de describir una muestra clinica de mujeres en base al tipo de alteraciones psicopatológicas que presentan, objetivo este que nos llevó a adoptar un planteamiento metodológico de tipo epidemiológico descriptivo, fundamentado en el diagnóstico de una serie de trastornos mentales a partir de un modelo de entrevista clínica totalmente estructurado, el DIS-III-R (Modelo de Entrevista de Diagnóstico, tercera versión revisada) (Robins, Helzer, Cottler y Goldring, 1989), el cual está basado a su vez en los criterios diagnósticos del DSM-III-R (Manual Diagnóstico y Estadístico de los Trastornos Mentales, tercera edición revisada) (APA, 1988).

Así, y dado que nuestra investigación y los resultados obtenidos de ella están basados en un concreto sistema de clasificación de los trastornos mentales (DSM-III-R) consideramos conveniente, aunque sin profundizar en ellos, destacar algunos aspectos teóricos concernientes al problema de la clasificación y diagnóstico en psicopatología así como a la situación actual acerca del estudio de la psicopatología en la mujer, antes de pasar a detallar las caracteristicas concretas del trabajo empírico realizado.

En lo que respecta a la primera de las cuestiones mencionadas -la clasificación y diagnóstico- todos conocemos la existencia de múltiples intentos de clasificación de las alteraciones de la conducta, acordes en cada momento con los valores y avances científicos de cada época. Sin embargo, uno de los más importantes avances en lo que respecta a la clasificación y diagnóstico de los trastornos mentales fue la publicación, en 1980 por la Asociación Americana de Psiquiatria, de la $3^{\mathrm{a}}$ edición del DSM (el DSM-III), ya que este sistema clasificatorio incorporaba importantes avances metodológicos tales como el uso de criterios diagnósticos específicos (de inclusión y exclusión) para una gran cantidad de categorias diagnósticas (150) o el uso de una clasificación multiaxial (con cinco posibles ejes). La revisión del DSM-III (DSM-III-R), publicada sólo siete años después (1987), propone numerosas modificaciones sobre el anterior, aunque relativamente menores, ya que únicamente persiguen corregir imperfecciones técnicas y lógicas del anterior manual. Puesto que este ha sido uno de los sistemas clasificatorios $y$ diagnósticos de entre los vigentes hasta la actualidad (aunque en 1994 fue publicado el DSM-IV), en él nos hemos basado para realizar nuestra investigación en base a la administración de una entrevista totalmente estructurada derivada de él, el DIS-III-R (Modelo de Entrevista de Diagnóstico, $3^{\mathrm{a}}$ versión revisada) (Robins et al., 1989), del que hablaremos más detenidamente cuando expongamos los aspectos metodológicos de nuestro trabajo.

Por otra parte, y en lo que concierne al estudio de la salud mental de la mujer y sus características distintivas, la investigación epidemiológica llevada a cabo en las últimas décadas ha demostrado, de manera inequivoca, la existencia de marcadas diferencias en la morbilidad psicopatológica y en el patrón de conducta de enfermedad desarrollados según el sexo. Este hecho se ha confirmado en estudios realizados tanto en poblaciones hospitalarias, como en pacientes que contactan con los consultorios de medicina general, como en la población general y ha sido verificado, asimismo, en nuestro propio pais (Vázquez-Barquero et al., 1986; Herrera et al., 1987, 1990).

Los mecanismos que se han utilizado para explicar estas diferencias pertenecen a dos tipos de formulaciones teóricas: biologicistas y ambientalistas. Las primeras establecen que es constitucional, lo genético y/o los factores endocrinos son los que condicionan las diferencias de morbilidad psicopatológica según el sexo, si bien los estudios realizados en esta área se muestran insuficientes para explicar el distinto comportamiento de cada uno de los sexos frente a la enfermedad mental (Weissman y Klerman, 1977; Togersen, 1983; Gershow, 1976; Frankenhauser, 1976). En el polo opuesto, las teorias ambientalistas defienden que son las variables socioculturales las que mejor explican las variaciones en la morbilidad y en los patrones de conducta de enfermedad encontrados. De esta forma, factores como el estado civil, la presencia de hijos menores en el hogar, o el estado ocupacional serian los responsables, actuando a través de los roles impuestos socialmente a cada sexo, del modo en que cada uno de ellos manifiesta su sufrimiento psicológico y de las 
estrategias que adopta para satisfacer sus necesidades de atención psiquiátrica (Brown y Harris, 1978; Bebbington, Hurry Tennant, Sturt y Wing, 1981). No obstante, los distintos estudios realizados no se muestran concluyentes en uno u otro sentido, lo cual apunta a la idea de que, posiblemente, dichas diferencias estén explicadas en mayor o menor medida por ambos tipos de factores.

La Tabla 1 describe, haciendo referencia a algunos aspectos metodológicos, la prevalencia psiquiátrica más relevante encontrada en ambos sexos en algunos de los estudios comunitarios realizados en la última década en España y otros paises. Observamos, en primer lugar, en las mujeres de todas las comunidades investigadas, unas altas cifras de alteraciones psicopatológicas, situándose la preva- lencia/mes entre el 12.4 y el $34.2 \%$; en segundo término destaca que dicha prevalencia es de manera constante significativamente más alta que la encontrada en el sexo masculino $y$, finalmente, que las cifras de morbilidad detectadas dependen, en alguna medida, del instrumento de exploración psicopatológica utilizado. Asi, la utilización de un cuestionario como el GHQ (General Health Questionnaire) (Goldberg y Williams, 1988) genera cifras de morbilidad muy uniformes, pero más altas que las obtenidas mediante entrevistas psiquiátricas como el PSE (Present State Examination) (Wing, Cooper y Sartorius, 1974) y el DIS.

El análisis de los estudios comunitarios que analizan la problemática de la comparación entre morbilidad psicopatológica femenina y masculina mediante el PSE o el CIS (Clinical

Tabla 1.- Prevalencia psiquiátrica encontrada en distintos estudios comunitarios realizados en España, y otros paises, según el sexo.

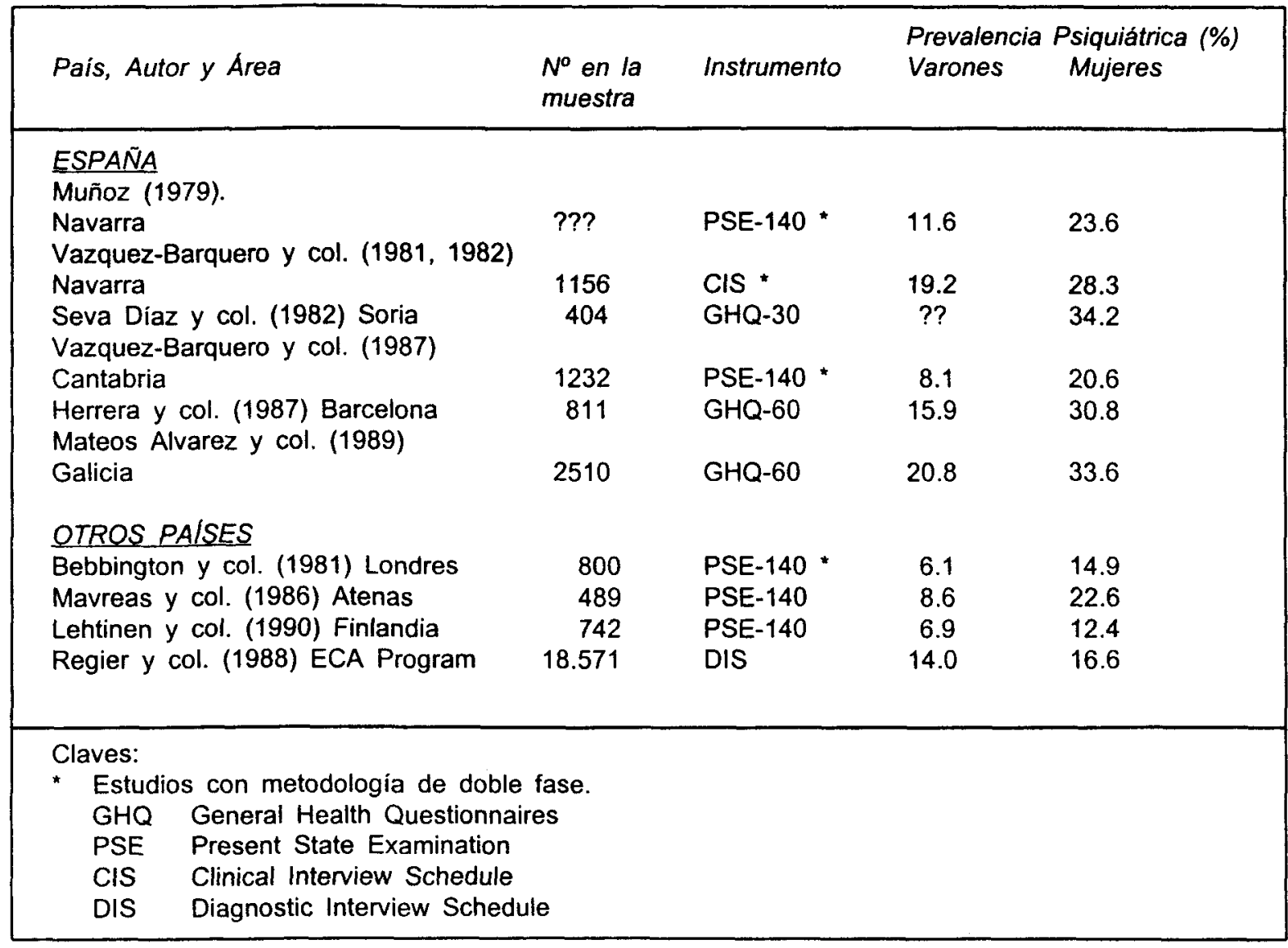


Interview Schedule) (Goldberg, Cooper, Eastwood, Kedward y Shepherd, 1970) revela que las mujeres presentan un exceso de morbilidad que se sitúa en torno a una proporción 2:1 con respecto a los hombres (ver Tabla 1). A similares conclusiones se llega en la Encuesta Nacional de Salud (Ministerio de Sa-nidad y Consumo, 1989) en la que se demuestra que el riesgo relativo de presentar alteraciones mentales es superior en el sexo femenino, ascendiendo la razón de tasas mujer/varón del 1.6 en el grupo de edad 16-44 años al 2.0 en las edades superiores a los 44 años. Dicho exceso está generado por la presencia de cifras de prevalencia significativamente más altas en los cuadros depresivos, de ansiedad y fóbicos.

Similar predominio femenino de morbilidad psicopatológica puede ser evidenciado en el proyecto ECA (Regier, Boy y Burke, 1988), si bien en este caso, el diferencial mujer/hombre se reduce considerablemente $(16.6 \% / 14.0 \%)$. Esto es debido a que, mientras que la depresión y la ansiedad son más comunes en las mujeres, otros trastornos como el alcoholismo, las adicciones o determinadas alteraciones de la personalidad lo son en los varones. Se podria por ello concluir que, lo realmente importante en las diferencias mujer/varón con respecto a la enfermedad mental no son las cifras de morbilidad global que ambos sexos presentan, sino más bien las formas psicopatológicas a través de las que se expresa dicha morbilidad.

\section{METODOLOGÍA}

\section{Objetivos}

El objetivo planteado al inicio de este trabajo, que será el que marque el desarrollo empírico de nuestra investigación, es el conocer la frecuencia de aparición de las distintas alteraciones psicopatológicas en una muestra clinica de mujeres, mediante la administración de un concreto instrumento de evaluación.

\section{Material y métodos}

\subsection{Descripción de las variables estudiadas}

Hemos sometido a estudio 40 variables referidas a los 40 posibles diagnósticos obtenidos en la muestra a partir de una entrevista estructurada (DIS) derivada del DSM-III-R. Dichas variables incluyen:

- Tres trastornos somatoformes

- Nueve trastornos por ansiedad

- Quince posibles trastornos del estado de ánimo

- Ocho trastornos por uso de sustancias psicoactivas

- Dos trastornos de la conducta alimentaria

- Esquizofrenia

- Trastorno antisocial de la personalidad

- Trastorno mental orgánico

\subsection{Instrumentos de medida}

La técnica de evaluación utilizada ha sido el instrumento de diagnóstico propiamente dicho, el "Modelo de Entrevista de Diagnóstico, tercera versión revisada" (Diagnostic Interview Schedule, third edition revised) (DIS-IIIR) (Robins et al., 1989). EI DIS (Diagnostic Interview Schedule, DIS) (Robins, Helzer, Croughan y Rataliff, 1981) es una entrevista psicodiagnóstica altamente estructurada que se creó a partir de los criterios del DSM-III, con la finalidad de ser utilizada en estudios epidemiológicos de campo de gran escala (Robins et al., 1981a). Una de sus características más singulares, impuesta por el diseño epidemiológico para el cual se concibió (estudio de muestras muy numerosas), es la de poder ser utilizada por entrevistadores sin experiencia en psicodiagnóstico clínico, tras solo un corto periodo de aprendizaje de varias semanas.

EI DIS es un instrumento complejo y extenso, que podemos catalogar dentro de la familia de entrevistas estructuradas/informatizadas (de Pablo, Albacete y Meroño, 1992), en el cual, cada pregunta o varias preguntas, son “alternativas" fijas y precodificadas de respuesta, que se hallan vinculadas a un criterio diagnóstico; su conjunto, lógicamente ordenado, permite que el total de la información obtenida una vez finalizada la entrevista, pueda traducirse a diagnósticos mediante una serie lógica de reglas de decisión. En 1989, Robins y cols., elaboraron la versión III-R del DIS, la cual incorpora cuestiones para que los diagnósticos puedan realizarse según el sistema DSMIII-R, y ésta ha sido la versión utilizada en nuestra investigación. 


\subsection{Descripción de la muestra}

En un primer momento de nuestra investigación, y en un intento de delimitar el proyecto, nos planteamos trabajar con un área concreta dentro de la población general de mujeres, referida a población clínica. Así, la muestra se ha obtenido de entre las mujeres que acuden a la/s consultas de Psiquiatria y Psicología del Hospital Virgen de la Arrixaca de Murcia, con edades comprendidas entre los 20 y los 55 años.

Obtuvimos de esta forma 76 protocolos, recogidos en un espacio de cinco meses (Febrero-Junio de 1994), que son los que han configurado la muestra definitiva.

\subsection{Procedimiento estadístico}

Una vez que dispusimos de los 76 protocolos que formarian la muestra, los datos de las 40 variables referidas a posibles diagnósticos se sometieron a un análisis exploratorio mediante el paquete estadistico SYSTAT (The System for Statistics), en versión 5.0 a partir de Ato y López-Garcia (1994), obteniendo las distribuciones de frecuencias y porcentajes de cada una de las variables.

\section{RESULTADOS}

Resumiremos en este momento los resultados obtenidos a través del análisis de las 40 variables referidas a posibles diagnósticos, analizando la frecuencia de aparición de éstos en el total de la muestra. A continuación, y puesto que una gran proporción de las mujeres son diagnosticadas de más de un trastorno, describiremos la muestra en función de que en cada una de sus componentes se haya realizado un solo diagnóstico (diagnóstico único) o más de uno (diagnóstico múltiple), respetando en este último caso los criterios de diagnósticos excluyentes marcados por el DSM-III-R.

\section{A) Análisis frecuencial de diagnósticos}

Tal y como se puede observar en las Tablas 2, 3 y 4 (y Figuras 1, 2 y 3) vemos que la mayor parte de los trastornos diagnosticados en nuestra muestra se agrupan en tres grandes categorias: Trastornos Somatoformes, Trastornos por Ansiedad y Trastornos del Estado de Animo, apareciendo el resto de diagnósticos en muy bajas proporciones (Tablas 5 y 6 ). En todos los casos, hablaremos siempre de la frecuencia relativa de trastornos con respecto al $100 \%$ de la muestra.

En lo que respecta a los Trastornos Somatoformes, solo una de las pacientes es diagnosticada de Trastorno por Somatización, mientras que $51(67.11 \%)$ lo son de Dolor Somatoforme y 34 ( $44.74 \%$ ) pertenecen a una categoria que, aunque no aparece como tal en el DSM-III-R, hemos considerado interesante introducir y que es la de "Trastorno por Somatización ausente, pero presentes seis o más síntomas a lo largo de la vida". No es posible obtener el porcentaje global de mujeres que presentan un Trastorno Somatoforme, ya que estos diagnósticos no son mutuamente excluyentes, y se produce un solapamiento al aparecer pacientes con más de uno de estos diagnósticos.

Lo mismo ocurre en el caso de los Trastornos por Ansiedad, en donde nos volvemos a encontrar un solapamiento de los distintos diagnósticos. No obstante, analizando parcialmente los resultados, observamos que un $22.37 \%$ de la muestra (17 pacientes) presenta un Trastorno por Angustia, siendo más frecuente con Agorafobia que sin ella; un $75 \%$ presenta algún tipo de Fobia, siendo la más frecuente la Fobia Social, y un $11.85 \%$ (9 pacientes) presenta un Trastorno Obsesivo-Compulsivo. Finalmente, podemos observar que solo tres pacientes de nuestra muestra son diagnosticadas de Trastorno por Ansiedad Generalizada.

Por último $\mathrm{y}$, en lo que respecta a los Trastornos del Estado de Animo, si es posible obtener el porcentaje total de la muestra que presenta un trastorno de este tipo ya que, en este caso, los diagnósticos si son mutuamente excluyentes. Observamos asi, que un $81.9 \%$ de la muestra (63 del total de 76 pacientes) presenta un trastorno del estado de ánimo, porcentaje global que se distribuye entre un $74.01 \%$ ( 57 mujeres) que presenta un trastorno de tipo depresivo, y solo un $7.89 \%$ (6 pacientes) que presenta un trastorno de tipo bipolar o un episodio hipomaniaco. Vemos que son, por tanto, mucho más frecuentes los trastornos de tipo depresivo $y$, dentro de éstos, predominan los que se presentan sin sintomas psicóticos sobre los que aparecen con dichos sintomas. 
Tabla 2.- Frecuencia Trastornos Somatoformes

\begin{tabular}{|lccc|}
\hline & Frecuencias & Porcentajes & Totales \\
\hline TRASTORNOS SOMATOFORMES & 1 & & \\
T. por Somatización & $1.32 \%$ & \\
T. por somatización ausente pero presentes seis & 34 & $44.74 \%$ \\
O más sintomas & 51 & $67.11 \%$ & \\
Dolor Somatoforme & $51 \%$ \\
\hline
\end{tabular}

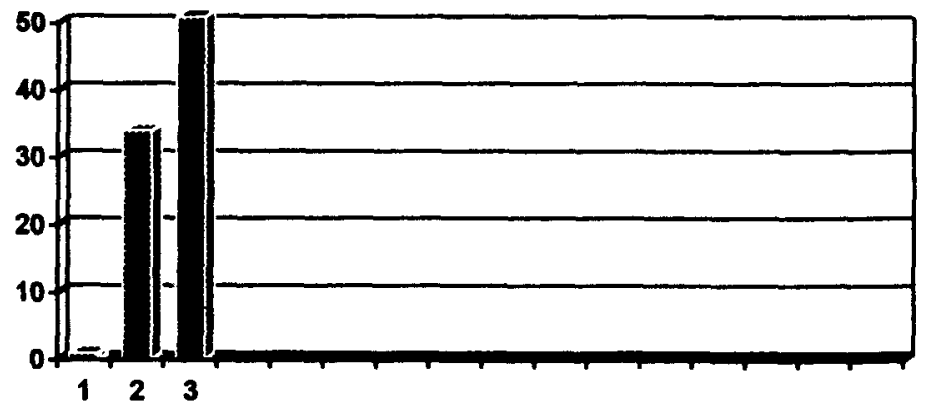

1. Trastorno por Somatización

3. Dolor Somatoforme

2. Trastomo por Somatización ausente pero presentes seis o más síntomas

Figura 1.- Frecuencia Trastornos Somatoformes

Tabla 3.- Frecuencia Trastornos por Ansiedad

\begin{tabular}{|lccc|}
\hline & Frecuencias & Porcentajes & Totales \\
\hline TRASTORNOS POR ANSIEDAD & & & \\
T. por angustia sin agorafobia & 5 & $6.58 \%$ & \\
T. por angustia con agorafobia & 12 & $15.79 \%$ & $17(22.37 \%)$ \\
Agorafobia sin angustia & 13 & $17.11 \%$ & $57(75 \%)$ \\
Fobia social & 23 & $30.26 \%$ & \\
Fobia simple & 21 & $27.63 \%$ & \\
T. obsesivo-compulsivo & 3 & $3.95 \%$ & \\
(obsesiones y compulsiones & 3 & $3.95 \%$ & $9(11.85 \%)$ \\
T. obsesivo-compulsivo (solo obsesiones) & 3 & $3.95 \%$ & \\
T. obsesivo-compulsivo (solo compulsiones) & 3 & $3.95 \%$ & \\
T. por ansiedad generalizada & 3 & & \\
\hline
\end{tabular}

1. T. por Angustia sin Agorafobia 2. T. por Angustia con Agorafobia

3. Agorafobia sin Angustia

5. Fobia Simple
4. Fobia Social

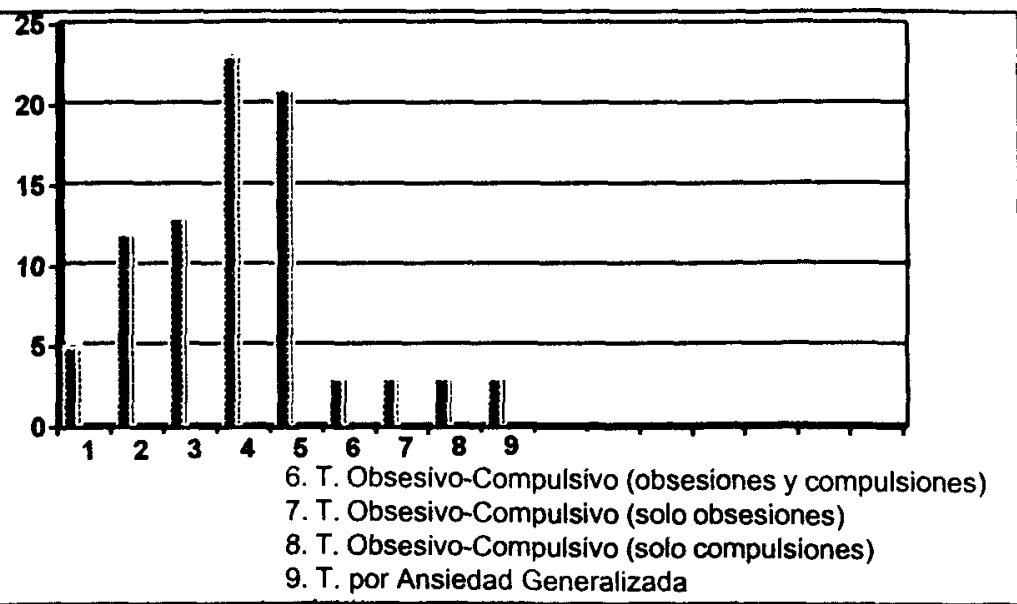

Figura 1.- Frecuencia Trastornos por Ansiedad 
Tabla 4.- Frecuencia Trastornos del Estado de Animo

\begin{tabular}{|c|c|c|c|}
\hline & Frecuencias & Porcentajes & Totales \\
\hline $\begin{array}{l}\text { TRASTORNOS DEL } \\
\text { ESTADO DE ANIMO } \\
\text { Episodio depresivo mayor }\end{array}$ & & & \\
\hline $\begin{array}{l}\text { con sintomas psicóticos } \\
\text { Episodio depresivo mayor }\end{array}$ & 0 & $0.00 \%$ & \\
\hline sin sintomas psicóticos & 1 & $1.32 \%$ & $1(1.32 \%)$ \\
\hline $\begin{array}{l}\text { Episodio hipomaniaco } \\
\text { Depresión mayor, episodio único }\end{array}$ & 2 & $2.63 \%$ & \\
\hline $\begin{array}{l}\text { con sintomas psicóticos } \\
\text { Depresión mayor, episodio único }\end{array}$ & 5 & $6.58 \%$ & \\
\hline $\begin{array}{l}\sin \text { sintomas psicóticos } \\
\text { Depresión mayor recurrente }\end{array}$ & 12 & $15.79 \%$ & $17(22.37 \%)$ \\
\hline $\begin{array}{l}\text { con síntomas psicóticos } \\
\text { Depresión mayor recurrente }\end{array}$ & 5 & $6.58 \%$ & \\
\hline sin síntomas psicóticos & 17 & $22.37 \%$ & $22(28.95 \%)$ \\
\hline $\begin{array}{l}\text { Distimia } \\
\text { T. depresivo no especificado }\end{array}$ & 2 & $2.63 \%$ & \\
\hline $\begin{array}{l}\text { con síntomas psicóticos } \\
T \text {. depresivo no especificado }\end{array}$ & 2 & $2.63 \%$ & \\
\hline sin sintomas psicóticos & 5 & $6.58 \%$ & $7(9.21 \%)$ \\
\hline T. bipolar con sintomas psicóticos & 2 & $2.63 \%$ & \\
\hline $\begin{array}{l}\text { T. bipolar sin sintomas psicóticos } \\
\text { Episodio depresivo mayor }\end{array}$ & 0 & $0.00 \%$ & $2(2.63 \%)$ \\
\hline $\begin{array}{l}\text { excepto criterios de exclusión } \\
\text { E. depresivo mayor y } E \text {. maniaco }\end{array}$ & 3 & $3.95 \%$ & \\
\hline excepto criterios de exclusión & 2 & $2.63 \%$ & \\
\hline Sintomatologia depresiva (leve) & 5 & $5.58 \%$ & \\
\hline
\end{tabular}

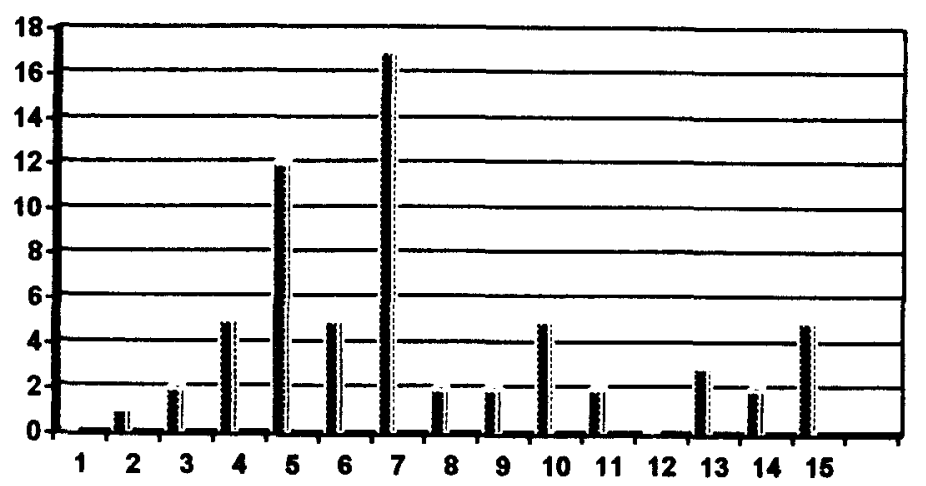

1. Episodio Depresivo Mayor con sintomas psicóticos

2. Episodio Depresivo Mayor sin sintomas psicóticos

3. Episodio Hipomaniaco

4. Depresión Mayor, episodio único con sintomas psicóticos

5. Depresión Mayor, episodio único sin sintomas psióticos

6. Depresión Mayor recurrente, con sintomas psicóticos

7. Depresión Mayor recurrente, sin sintomas psicóticos

8. Distimia
9. T. Depresivo no especificado con síntomas psicóticos 10. T. Depresivo no especificado sin sintomas psicóticos 11. T. Bipolar con síntomas psicóticos

12. T. Bipolar sin sintomas psicoticos

13. Episodio Depresivo Mayor excepto criterios de exclusión 14. E. Depresivo Mayor y E. Maniaco excepto criterios de exclusión 15. Sintomatologia depresiva (leve)

Figura 3.- Frecuencia Trastornos del Estado de Ánimo 
Tabla 5.- Frecuencia Trastornos por Uso de Sustancias Psicoactivas

\begin{tabular}{|c|c|c|c|}
\hline & Frecuencias & Porcentajes & Totales \\
\hline \multicolumn{4}{|l|}{$\begin{array}{l}\text { TRASTORNOS POR USO DE } \\
\text { SUSTANCIAS PSICOACTIVAS }\end{array}$} \\
\hline Dependencia de alcohol leve & 0 & $0.00 \%$ & \\
\hline Dependencia de alcohol moderada & 0 & $0.00 \%$ & \\
\hline Dependencia de alcohol grave & 0 & $0.00 \%$ & \\
\hline Abuso de alcohol & 0 & $0.00 \%$ & \\
\hline Dependencia de drogas prescritas leve & 2 & $2.63 \%$ & \\
\hline Dependencia de drogas prescritas moderada & $\overline{0}$ & $0.00 \%$ & \\
\hline Dependencia de drogas prescritas grave & 0 & $0.00 \%$ & \\
\hline Abuso de drogas prescritas & 0 & $0.00 \%$ & \\
\hline
\end{tabular}

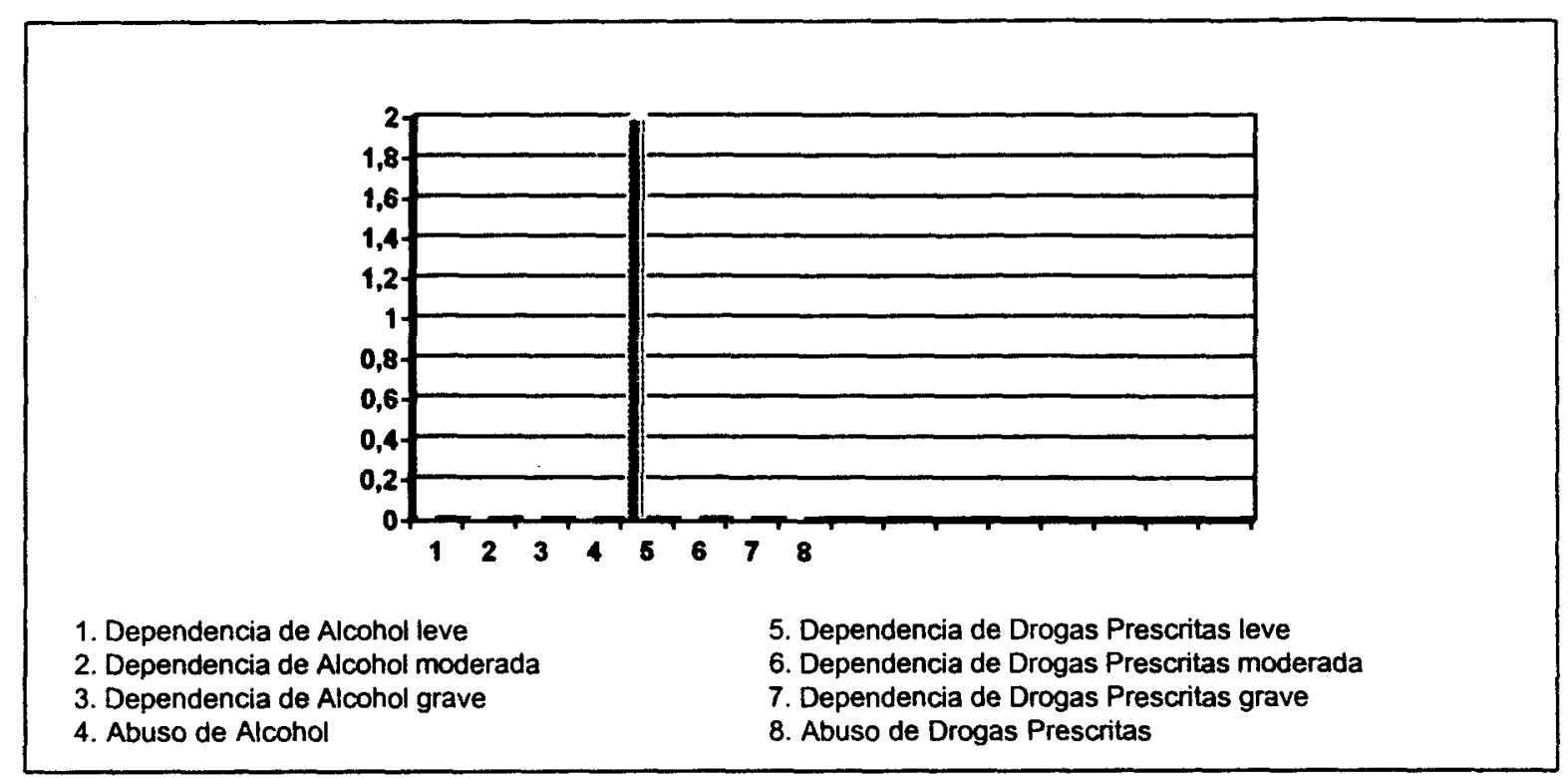

Figura 4.- Frecuencia Trastornos por Uso de Sustancias Psicoactivas

En las tablas y figuras siguientes se pueden observar los resultados referidos al resto de posibles diagnósticos sometidos a estudio (Trastornos por uso de sustancias psicoactivas, Trastornos de la conducta alimentaria, Esquizofrenia, Trastorno antisocial de la personalidad y Trastornos mentales orgánicos), constatándose en todo caso la baja frecuencia de aparición de éstos en el total de la muestra: un $2.63 \%$ de ésta ( 2 pacientes) presenta una Dependencia de drogas prescritas (leve), un $2.63 \%$ recibe el diagnóstico de Bulimia nerviosa y sólo una de las 76 pacientes (1.32\%) está diagnósticada de Esquizofrenia.

\section{B) Análisis frecuencial diagnósticos únicos y múltiples}

Ante la observación de que una gran proporción de mujeres reciben más de un diag- nóstico, ofreceremos de forma resumida (Tablas 7 y 8/Figura 6) una caracterización global de la muestra en función de las tres grandes categorias de trastornos que se han mostrado como más frecuentes en nuestro estudio (Trastornos somatoformes, Trastornos por ansiedad y Trastornos del estado de ánimo) y las posibles combinaciones en las que aparecen en las 76 mujeres.

Como se puede observar en dichas tablas, hemos agrupado a las mujeres en función de que se encuentren incluidas en una sola de esas tres categorias de trastornos anteriormente mencionadas, o en cada una de las tres posibles combinaciones entre ellas: Trastornos somatoformes y por ansiedad, Trastornos somatoformes y del estado de ánimo y Trastornos por ansiedad y del estado de ánimo. Asimismo, hemos obtenido el porcentaje de 
Tabla 6.- Frecuencia otros trastornos

\begin{tabular}{|c|c|c|c|}
\hline & Frecuencias & Porcentajes & Totales \\
\hline \multicolumn{4}{|l|}{$\begin{array}{l}\text { TRASTORNOS DE LA } \\
\text { CONDUCTA ALIMENTARIA }\end{array}$} \\
\hline Anorexia nerviosa & 0 & $0.00 \%$ & \\
\hline Bulimia nerviosa & 2 & $2.63 \%$ & \\
\hline $\begin{array}{l}\text { ESQUIZOFRENIA } \\
\text { TRASTORNO ANTISOCIAL }\end{array}$ & 1 & $1.32 \%$ & \\
\hline $\begin{array}{l}\text { DE LA PERSONALIDAD } \\
\text { TRASTORNOS MENTALES }\end{array}$ & 0 & $0.00 \%$ & \\
\hline ORGANICOS & 0 & $0.00 \%$ & \\
\hline
\end{tabular}

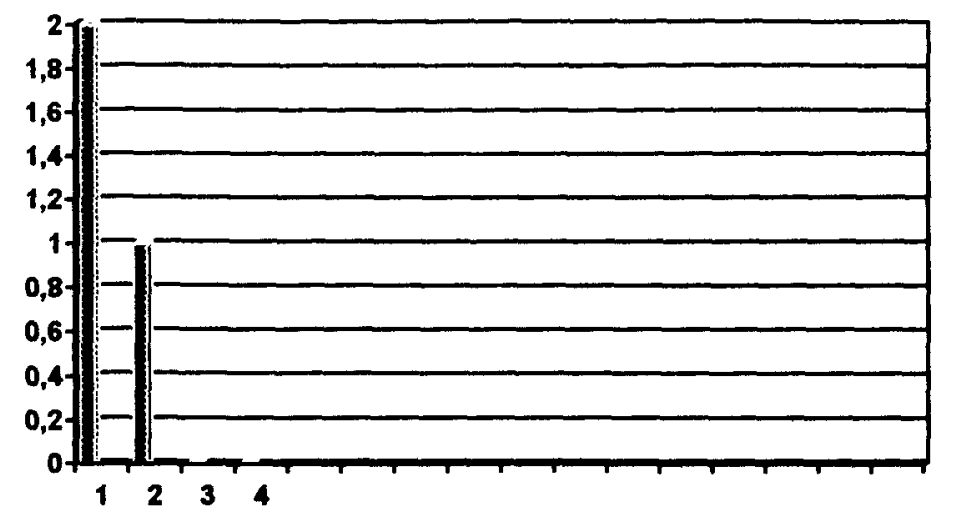

1. Trastomos de la Conducta Alimentaria (Bulimia)

3. Trastorno Antisocial de la Personalidad

2. Esquizofrenia

4. Trastornos Mentales Orgánicos

Figura 5.- Frecuencia otros trastornos

Tabla 7.- Frecuencia Diagnósticos Únicos

\begin{tabular}{|lcrc|}
\hline & Frecuencias & Porcentajes & $\begin{array}{l}\text { \%Totales } \\
\text { por grupos }\end{array}$ \\
\hline DIAGNÓSTICOS ÚNICOS & & & \\
Trastornos Somatoformes & 7 & 9.21 & $19(25 \%)$ \\
Trastornos por Ansiedad & 4 & 5.26 & \\
Trastornos del Estado de Animo & 8 & 10.52 & \\
\hline
\end{tabular}

mujeres que reciben diagnósticos de las tres categorias y el de mujeres que no reciben ningún diagnóstico.

Pasamos a detallar la información contenida en dichas tablas:

1) En primer lugar (Tabla 7), observamos que solo 19 de nuestras mujeres $(25 \%)$ reciben diagnósticos que se incluyen en una sola categoria: ocho de ellas en la de Trastornos del estado de ánimo (10.52\%), siete en la de Trastornos somatoformes $(9.21 \%)$ y solo cuatro en la de Trastornos por ansiedad (5.26\%).

2) En segundo lugar (Tabla 8), cabe resaltar que una proporción elevada de mujeres $(32.89 \%)$, en concreto 25 de las 76 mujeres estudiadas, recibe al mismo tiempo diagnósticos pertenecientes a dos categorias y, lo que es más importante, la gran mayoria de ellas, 
Tabla 8.- Frecuencia Diagnósticos Múltiples

\begin{tabular}{|lccc|}
\hline & Frecuencias & Porcentajes & $\begin{array}{l}\text { \% Totales } \\
\text { por grupos }\end{array}$ \\
\hline DIAGNÓSTICOS MÚLTIPLES & & & \\
Trastornos Somatoformes y por Ansiedad & 3 & 3.94 & \\
Trastornos Somatoformes y del Estado de Animo & 18 & 23.68 & $25(32.89 \%)$ \\
Trastornos por Ansiedad y del Estado de Animo & 4 & 5.26 & \\
LOS tres tipos de trastornos & 30 & 39.47 & $30(39.47 \%)$ \\
NINGÚN TRASTORNO & 2 & 2.63 & $2(2.63 \%)$ \\
& & & \\
\hline
\end{tabular}

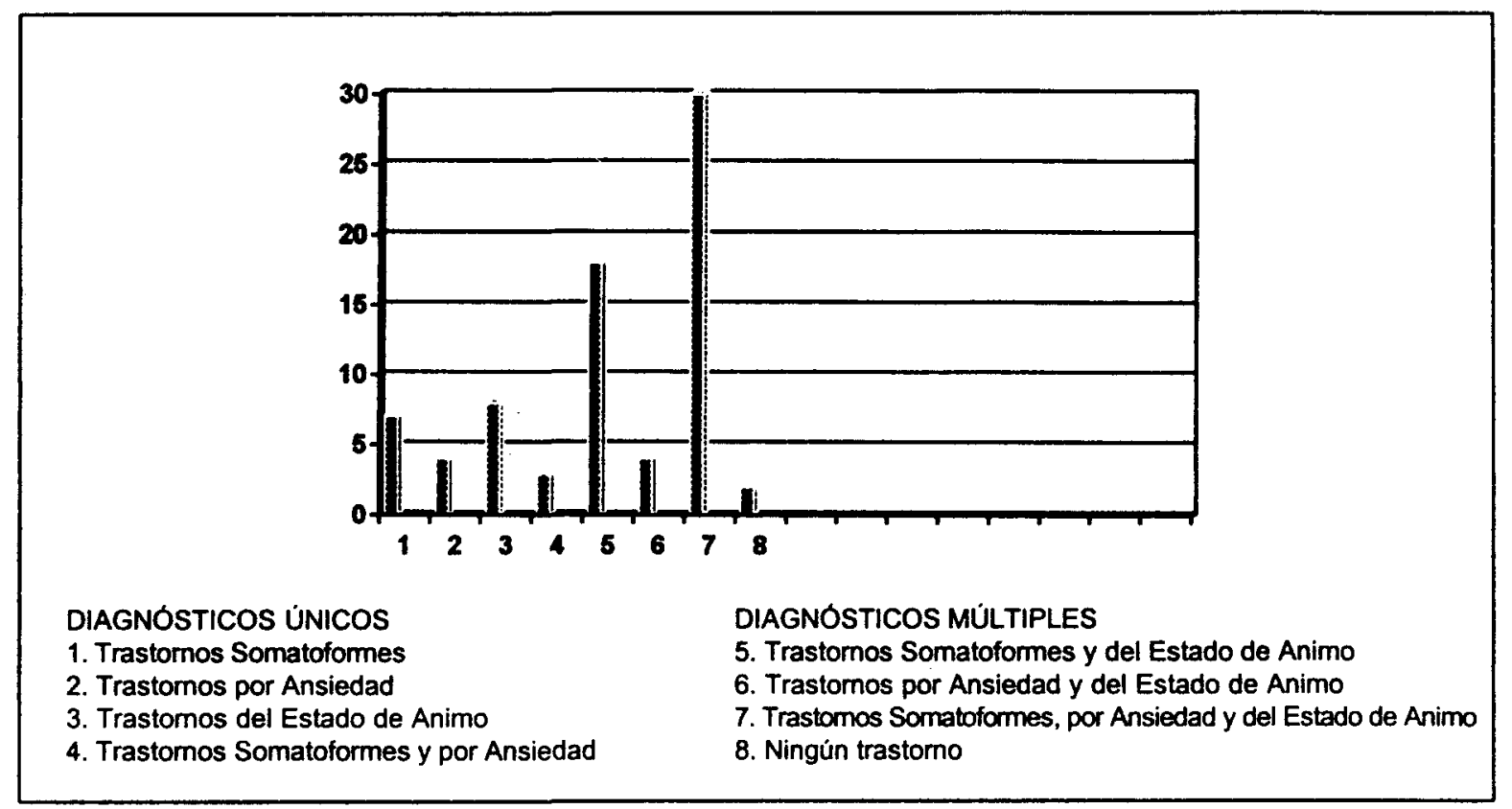

Figura 6- Frecuencia Diagnósticos Únicos y Múltiples

un $23.68 \%$ (18 mujeres) presenta conjuntamente algún Trastorno somatoforme y del estado de ánimo.

3) En tercer lugar (Tabla 8), es especialmente llamativo que un $39.47 \%$ de la muestra, lo que supone un total de 30 mujeres, presenta diagnósticos encuadrables a un tiempo en las tres grandes categorias.

En definitiva $y$, haciendo una valoración global, mientras que solo un $25 \%$ de nuestra muestra recibe diagnósticos pertenecientes a una sola de las tres grandes categorias, una amplísima proporción, el $72.36 \%$ (55 mujeres), presenta trastornos en más de una categoria, y solo el $2.63 \%$ (dos mujeres) no pre- senta, ni en la actualidad ni en el pasado, ningún tipo de sintomatología con significación clínica.

\section{CONCLUSIONES}

Las conclusiones derivadas de los resultados obtenidos y que intentarian responder al objetivo que nos planteábamos al inicio de la investigación en relación a la morbilidad psicopatológica, se podrian concretar en los siguientes puntos:

Se observa en nuestra muestra un claro predominio de tres grandes categorias de trastornos: trastornos del estado de ánimo, tras- 
tornos por ansiedad y trastornos somatoformes (tal y como se confirma en estudios como los de Lehtinen, Lindholm, Veijola y Vaisanen, 1990; o Vázquez-Barquero et al., 1987).

Respecto a los trastornos por ansiedad, se confirma la prevalencia establecida en el DSMIII-R (APA, 1988) en el sentido de que en población clinica es mucho más común el Trastorno por Angustia con Agorafobia que sin ella, y que la Agorafobia sin Historia de Trastorno por Angustia es una alteración más rara que otras fobias como la Social o la Simple. Asimismo, corroboramos los datos de prevalencia del Trastorno Obsesivo-Compulsivo y Trastorno por Ansiedad Generalizada en el sentido de que son poco frecuentes en muestras clinicas (DSM-III-R).

En este punto consideramos necesario comentar los datos referidos a la fobia simple y social. En nuestra opinión, el elevado número de mujeres diagnosticadas de estos trastornos en nuestra muestra se debe a la baja rigurosidad que, creemos, presenta el DIS para estos trastornos ya que se evalúan mediante preguntas abiertas $y$, en cierto modo abstractas, que no especifican con claridad los sintomas de ansiedad que el paciente debe mostrar ante el estimulo o situación fóbica, con lo cual se le deja un gran margen para que valore subjetivamente si el miedo que siente es o no lo suficientemente intenso. Este hecho, desde nuestro punto de vista, ha aumentado considerablemente el número de diagnósticos de fobia realizado, ya que las pacientes han tendido a valorar como significativos unos temores que, en realidad, no son incapacitantes.

Respecto a los trastornos del estado de ánimo, de nuevo se confirman los datos sobre prevalencia de las Depresiones Mayores del DSM-III-R, que hablan de muy altas proporciones, especialmente en mujeres.

En relación a los trastornos somatoformes, se confirman los datos de prevalencia del DSM$I I I-R$, en el sentido de que es más frecuente la aparición de Dolor Somatoforme que de Trastorno por Somatización.

Confirmando de nuevo los resultados de los estudios revisados sobre diagnósticos múltiples (Vázquez-Barquero, Muñoz y Madoz Jauregui, 1981; Vázquez-Barquero, Peña, Diez Manrique, Arenal, Quintanal y Samaniego, 1988; Vázquez-Barquero, Wilkinson, Williams,
Díez Manrique y Peña, 1990), los trastornos somatoformes aparecen, en nuestras mujeres, asociados predominantemente a los trastornos del estado de ánimo, con clara superioridad sobre las asociaciones con los trastornos por ansiedad.

Por último, hemos de resaltar que, en nuestra muestra, es más frecuente el que en una misma persona aparezcan trastornos pertenecientes a más de una categoría que a una sola de ellas.

En resumen $y$, para terminar, consideramos que el objetivo que nos planteamos al inicio del presente trabajo se ha llevado a cabo y que las conclusiones obtenidas podrian ser un punto de partida para proseguir el estudio sobre la salud mental de las mujeres con el fin de intentar, en la medida de lo posible, analizar todas aquellas variables que pueden repercutir negativamente en dicha salud mental (temperamentales, socioculturales,...), porque conocer a fondo los posibles factores predisponentes y de riesgo es indispensable si pretendemos conseguir el fin último que es la prevención.

\section{REFERENCIAS BIBLIOGRÁFICAS}

American Psychiatric Association (1987). Diagnostic and statistical manual of mental disorders, third edition - revised (DSM-III-R). Washington, D.C.: APA.

American Psychiatric Association (1988). Manual diagnóstico y estadístico de los trastornos mentales, $3^{a}$ edición revisada (DSM-III-R). Barcelona: Masson.

Ato, M. y López-Garcia, J. (1994). Fundamentos de estadistica con SYSTAT. Madrid: Rama.

Brown, G.W. y Harris, T. (1978). Social origins of depression: a study os psychitric disorder in women. Londres: Tavistock.

Frankenhauser, M., Dunne, E. y Lundberg, V. (1976). Sex differences in sympathetic adrenal medullary reactions induced by different stresses. Psychopharmacology, 47, 1-5.

Gershow, E.S. y Bunney, W.E. (1976). The question of $X$-linkage in bipolar manic depressive illness. Journal of Psychiatry Research, 13, 99-117.

Goldberg, D. y Williams, P. (1988). A user's guide to the General Health Questionnaire. Windsor, Berkshire: Nfer-Nelson.

Goldberg, D., Cooper, B., Eastwood, M.R., Kedward, H.B. y Shepherd, M. (1970). A standardized 
psychiatric interview suitable for use in community surveys. The British Journal of Preventive Social Medicine, 24, 18-23.

Herrera, R., Antonell, J. Spagnolo, E. y Gispert, R. (1990). Estudio epidemiológico en salud mental de la comarca del Baix LLobregat (Barcelona). Informaciones Psiquiátricas, 120, 111130.

Herrera, R., Antonell, J. Spagnolo, E., Domenech, J. y Martín, S. (1987). Estudio epidemiológico en salud mental de la comarca del Baix LLobregat (Barcelona). Informaciones Psiquiátricas, 107.

Lehtinen, V., Lindholm, T., Veijola, J. y Vaisanen, E. (1990). The prevalence of PSE-CATEGO disorders in a finnish adult population cohort. Social Psychiatry and Psychiatric Epidemiology, 25, 187-192.

Muñoz, P.E. (1979). Estudio de la distribución de los trastornos psiquiátricos y de los niveles de salud mental en una población rural: aplicación de un método estándar al conocimiento de la morbilidad psiquiátrica. Madrid: Universidad Autónoma. Tesis Doctoral.

Pablo, F. de, Albacete, A., Meroño, A. y cols. (1992). Validez de la versión española del Diagnostic Interview Schedule. Tercera versión revisada (DIS-III-R). Actas Luso-españolas de Neurologia, Psiquiatria y Ciencias Afines, 20(6), 257 262.

Regier, D.A., Boy, J.H., Burke, Jr J.D. y cols. (1988). One-month prevalence of mental disorders in the United States. Based on five epidemiological catchment area sites. Archives of General Psychiatry, 45, 997-986.

Robins, L.A., Helzer, J.E., Croughan, J. y Rataliff, N. (1981b). National institute of mental health Diagnostic Interview Schedule: version II. NIMH, USA.
Robins, L.N., Helzer, J.E., Cottler, L. y Goldring, E. (1989). Diagnostic interview schedule; version III-R. Washington University Department of Psychiatry.

Togersen, S. (1983). Genetics of neurosis. The effect of sampling variation upon the twin concordance ratio. The British Journal of Psychiatry, 142, 126-132.

Vazquez-Barquero, J.L., Diez Manrique, J.F., Peña, C. y cols. (1987). A community mental health survey in Cantabria: a general description of morbidity. Psychological Medicine, 17, 227-241.

Vazquez-Barquero, J.L., Diez Manrique, J.F., Peña, C., Quintanal, R.G. y Labrador López, M. (1986). Two stage design in a community survey. The British Journal os Psychiatry, 149, 88-97.

Vazquez-Barquero, J.L., Muñoz, P.E. y Madoz Jauregui, V. (1981). The interaction between physical illness and neurotic morbidity in the community. The British Joumal of Psychiatry, 139, 328-335.

Vazquez-Barquero, J.L., Peña, C., Diez Manrique, J.F., Arenal, A., Quintanal, R.G. y Samaniego, C. (1988). The influence of sociocultural factors on the interaction between physical and mental disturbances in a rural community. Social Psychiatry and Psychiatric Epidemiology, 23, 195-201.

Vazquez-Barquero, J.L., Wilkinson, G., Williams, P. Díez Manrique, J.F. y Peña, C. (1990). Mental health and medical consultation in primary care settings. Psychological Medicine, 20, 681-694.

Weissman, M.M. y Klerman, G.L. (1977). Sex differences and the epidemiology of depression. Archives of General Psychiatry, 34, 98-111.

Wing; J.K., Cooper, J.E. y Sartorius, N. (1974). The measurement and clasiffication of psychiatric symptons. Nueva York: Cambridge University Press. 